ISSN 2693-2490

\title{
Considerations of Consciousness and Emotive Memory
}

Journal of Psychology and Neuroscience

Review Article

\author{
Gerard Marx $^{1 *}$, Chaim Gilon²
}

$\begin{array}{ll} & \text { *Correspondence author } \\ & \text { Gerard Marx } \\ { }^{1} M X \text { Biotech Ltd., Jerusalem, Israel } & \text { MX Biotech Ltd. } \\ & \text { Jerusalem } \\ { }^{2} \text { Institute of Chemistry, Hebrew University, Jerusalem, Isra } & \text { Israel }\end{array}$

Submitted : 20 Jun 2021 ; Published : 5 Jul 2021

\begin{abstract}
The role of chemistry is generally overlooked in theories of consciousness; most neuroscientists focus exclusively on electrodynamic signaling. We argue that chemodynamic signaling modes must also be considered.

As an aide to continuing this discourse, we clarify key terms, namely: Feelings, Emotions, Code and Neural net. In particular, we distinguish between "memory" as applied to the binary formatted "information" employed by computers, which lack any affective quality, and "emotive memory", the recall of subjective "cognitive information" experienced by neural nets.

Most concepts of consciousness focused on the electrodynamic activation, witness the many popular books and movies, as well as scientific papers based on this premise. However, the discovery of neurotransmitters (NTs) and development of psychoactive drugs indicates that consciousness is also enabled by chemodynamic processes, which particularly impact affective states. A graphic timeline is presented which highlights the historical milestones in the neuroscientific clarification of signaling modes pertinent to consciousness.

We opine that a combined chemodynamic and electrodynamic description of emotive memory will clarify the causative processes from which the experiential consciousness of the neural net emerges. Consider that without chemically encoded emotive memory, a conscious creature could not long survive; its consciousness would be moot.
\end{abstract}

Keywords : Cognition, Electrodynamic, Chemodynamic, Tripartite Mechanism, Emotive Memory, Neural Net.

\section{Background}

Scientists from various disciplines, such as physics, mathematics and computation, have tried to rationalize cognition (mentality) by linking considerations of consciousness to space, time and memory (Atkinson \& Shiffrin, 1965; Freeman, 1997; Arbib, 2000; Lange, 2002; Yin \& Troger, 2011; Sorli et al, 2016). Philosophers of various stripes also attempted to clarify the basis of consciousness (Morris, 1932; Ryle, 1949; Matzon, 1971; Chalmers, 1995). It has been argued that there must be a molecular perspective to the phenomenon of consciousness (Prentner, 2017).

A general critique of most approaches is that though they sometimes invoke psycho-physical processes, they do not offer a physiologically credible mechanism.

For example, Sorli et al provided a masterful review of the physics of space and time. However when they jumped to consciousness, their comments did not address the physiologic workings of a conscious neural net. Consider some quotes:

- "Consciousness ...can be described as n-dimensional

Hilbert space”.

- "Consciousness is the fundamental vibration of space".
- "Bio-photons are an information bridge between the atomic level and high dimensional Hilbert space."

- "A photon observed in a 3D world is connected with consciousness via Hilbert spaces”.

- "the energy of primordial consciousness in n-dimensional Hilbert space has infinite energy"

Their comments focused on mathematical mysteries but did not ascribe a causal relationship between the neural signaling and the emergence of a mental state.

Atkinson \& Shiffrin proposed mathematical algorithms and formulae to describe memory, a facet of consciousness (Figure 1). 


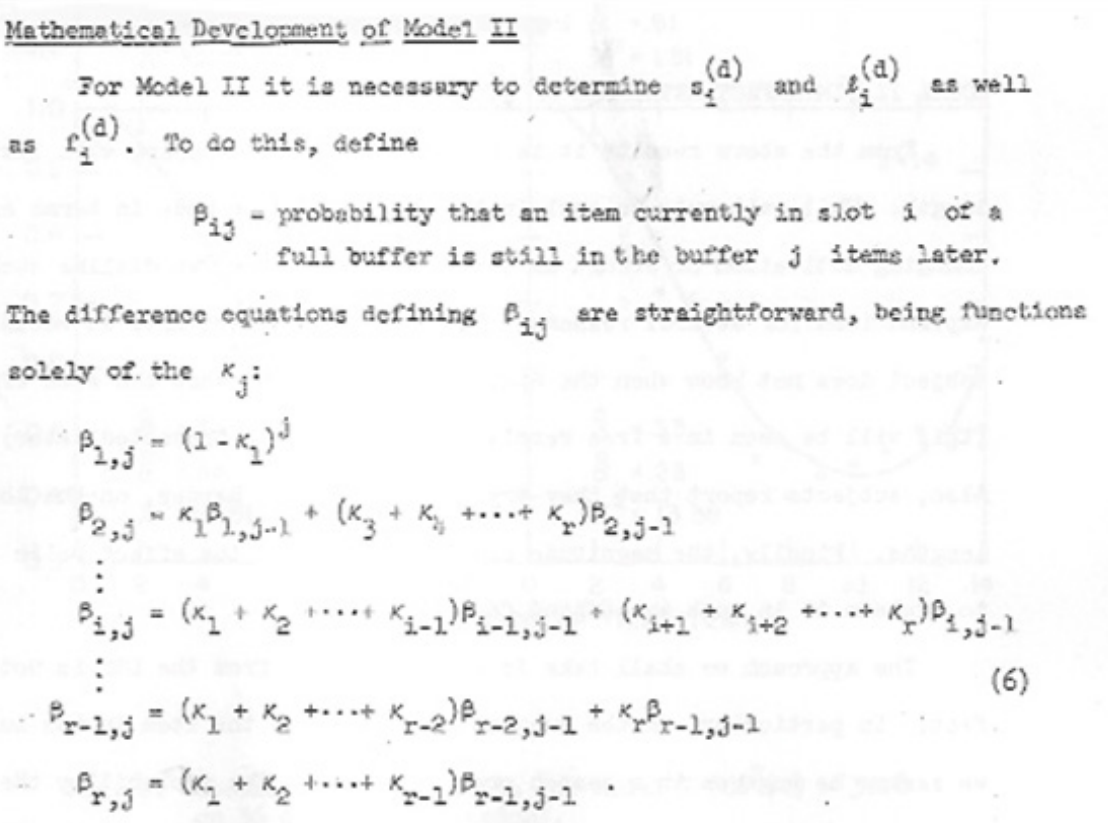

Figure 1: Some equations of the Atkinson-Shiffin (AS) mathematical model of human memory.

Others also suggested a mathematical approach to mentality (Sloman, 2008). What was missing in the mathematical treatment was a relevance to physiologic processes capable of encoding emotive memory.

Arbib reviewed the history of the concept of neural networks, particularly focusing on the work of McCullogh, whose work provided a compelling argument that thought (i.e. consciousness) could be expressed, simply through the interactions of neurons - without a mind or soul to influence the brain. Essentially, McCullogh and Pitts (1943) mathematically described sets of neurons which uni-directionally signaled in binary modes (Equation 1).

Equation 1:

$$
N_{i}\left(z_{1}\right) . \equiv . S\left\{\prod_{m=1}^{q} \sim N_{j m}\left(z_{1}\right) \cdot \sum_{a n i} \prod_{m a} N_{i o}\left(z_{1}\right)\right\}
$$

von Neumann, and Shanon attended McCullogh's lecture at the 1948 Hixon Symposium (Jeffress 1951). Though impressive, McCullogh's equations did not throw much light on biological neural mentation or the emergence of conscious states. Rather, their mathematical treatment helped establish the theory and practice of electronic microprocessors at the core of modern memory chips (von Neumann, 1951). Arbib suggested that McCullogh's "logical calculus" had great philosophical importance, it contributed more to the development of electronic devices than to neuroscience, as the electrical engineering approach could not formulate the emotive states that characterize the consciousness of neural creatures. Notwithstanding, the term "neural network" was appropriated by the gurus of Artificial Intelligence (AI) to refer to microprocessors hardwired with many connections (Franklin, 1995; Merolla et al. 2014), though these do not at all mimic the indirect (synaptic) contacts between biological neurons (more on this below).
Some workers deny a connection between neural activity and consciousness (Gerbaulet \& Henry, M. 2019), though this runs counter to clinical neuroscientific experience (see: anaesthetics). Others (Cleeremans, 2008) sketched out a conceptual framework rooted in "learning mechanisms" and "representational systems". However, these ideas did not relate to physiologic processes capable of enabling neural memory.

The quantum physicist Shrodinger also attended the 1948 Hixon Symposium and penned an essay entitled "Mind and Matter"(ShrodingerE. 1958). He asked: "What kind of material processes are directly associated with consciousness?" $\mathrm{He}$ embraced Spinoza's position of universal animation, that "any inanimate bodily thing is a thought of G-D" (Matson 1971). Though Shrodinger identified nervous processes as a form of rationalization and referred to Semon's concept of Meme (i.e. engram) (Semon, 1923), he was stumped by "unproved and unprovable speculation" regarding consciousness.

Rosenblum \& Kuttner (2011) described the solid science of classical physics as an expression of Quantum Mechanics (QM). At length, they discussed the 2-slit experiment as the archtypical demonstration of QM that "displays physics encounter with consciousness". They tried to resolve the enigma of consciousness by falling on the mysteries of QM (i.e. Shrodinger's 2-state cat). But their reach exceeded their grasp. Their review of physics suggested that Consciousness was an expression of neural net signaling. But they ignored neurophysiology and could not formulate how emotive states, an inelectable aspect of neural sensibility, could be achieved with QM formulations.

In a more biologically slanted approach, Yin \& Troger attempted to explain the neural basis of the recall (memory) by invoking interval timing, "a pattern of cortical activation with the stored memories", a logical tautology that leads nowhere. 
Churchland (2013) provided a more biologically credible discussion. That is, he began with the ontological problem (Descartes'mind-body puzzle (Skirry, 2016)) and reviewed the dualist and materialist theories of Mind. He discussed the difficulties of the dualist conviction in regard to mental states based on religious beliefs or suggestions of a "ghost in the machine" (Ryle, 1949). He waxed more enthusiastic with the materialist position, that all life processes are expressions of physico-chemical phenomena. He described neurons and discussed the evolution of neural nets and brains. However, Churchland's model was based on Cajal's "naked neuron" model (de Castro \& Merchán, 2016), ignoring the neural extracellular matrix (nECM) enshrouding the neurons through which electrical as well as chemical signals permeate (see tripartite mechanism discussed below).

Some attempted to credit consciousness to the organization of tubulin and microtubules which power movement and contractions of neural dendrites. These were considered to reflect coherent quantum processes in collections of microtubules within brain neurons, (Hameroff \& Penrose, 1995; Tuszynski 2006). However, the kinetics of tubulin assembly and disassembly argues against their role as a coding substrate for persistent emotive memory.
Edelman \& Tononi (2000) adopted a credible biologic approach. That is, they assumed that consciousness is a process that arises from the structure and dynamics of neural groups, which evolved with the increasing complexity of neural creatures. However, their "Picture of the Brain" (Chapter 4) was also based on Cajal's "naked neuron" model, ignoring the surrounding $\mathrm{nECM}$ as an active component of the neural signaling process that results in emotive memory and consciousness (see tripartite mechanism described below).

The hippocampus is a central feature of brain anatomy that is involved in memory and learning. A number of groups used immuno-imaging techniques on hippocampus slices to identify receptors to neurotransmitters (NTs) that are crucial to achieving affective states (Hörmera et al, 2018; Jong et al, 2018; Albrecht et al, 2021). Cumulatively, their work confirmed a critical role for the hippocampus in cognition, learning and emotion. They demonstrated that NT receptors are expressed by complementary neuronal populations of the dorsal hippocampus. They also pointed out that G proteincoupled receptors (GPCRs) play a dynamic role in synaptic plasticity, learning and memory. However, they did not propose a causal mechanism leading to mentality, or describe how NTreceptor interactions result in affective states characteristic of emotive memory and consciousness (Table 1).

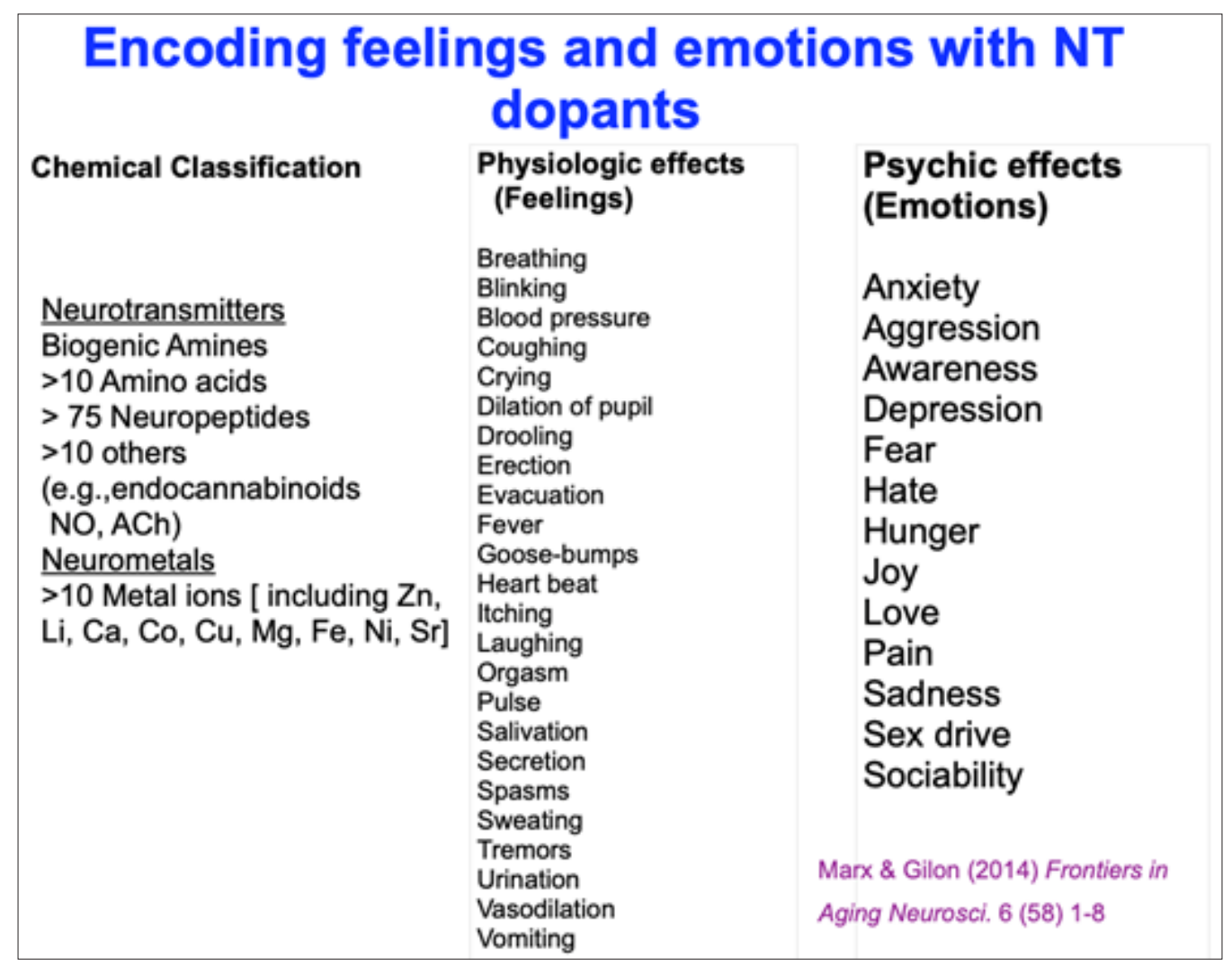


Changeux and coworkers (2010-2018) helped focus attention to the NT, acetylcholine, and its receptor (nAChR) which possesses all the structural elements required to convert a chemical signal into an electrical signal that causes an opening of ion channels. Thus, it behaves as a sophisticated, allosteric machine. They also focused on neuroimaging and electrophysiological signaling, contrasting human conscious and nonconscious processing. In particular, Changeux et al (2100) considered conscious processing of incoming information by modular brain systems (i.e. prefrontal, parieto-temporal and cingulate cortices) comprised of interconnected neurons with long-range axons with perceptual, motor, memory, evaluative, and attentional processors. They admitted that memory retrieval is an indispensable component of the more complex forms of self-awareness and consciousness However, they and other workers's broad approaches to consciousness were based on electrodynamic signaling between anatomic regions (D'Esposito, 2007). Such gross techniques could not provide resolution of how individual memories are encoded or merged into comprehensive consciousness. Moreover, they did not define the term "memory" which could apply either to an electronic device replay of stored information, or to the neural system's "emotive memory", as discussed below.

Robots, Cyborgs, Replicants and Artificial Intelligence A search of the term "consciousness" in YouTube or Wikipedia reveals that there is great popular interest in this subject, exemplified by the many lectures, presentations and articles, too numerous to cite here.

Computer scientists attempted to address the enigmas of consciousness by adopting the exclusive electrodynamic, binary signaling model proposed by Cajal and later generations of neuroscientists (McCullogh \& Pitts, 1942; Hebb, 1949; Bosse et al, 2014; Gallistel \& King, 2009). The field of Artificial Intelligence (AI) is rife with the assumption that binary algorithms could mimic the mentation talents of neural brains; that computation and mentation are equivalent processes. (Arbib, 1987; Franklin, 1995; Longuet-Higgins, 1981; Gardner, 1985; Sejnowski et al, 1988; Russel and Norvig, 2009; Aho, 2011; Guidolin et al, 2011; Howard, 2012; citing only a few).
This was rebuffed by authors who questioned the underlying premises of the computer model, notably the lack of emotive signifiers in the computer code (Arshavsky, 1993; Marx \& Gilon, 2018; Pockett, 2019).

\section{Merging Electrodynamic and Chemodynamic Signaling}

There are two historical streams in the conceptualization of neural processes resulting in consciousness; an electrodynamic mode and a chemodynamic mode. Most early attention focused on electrodynamic signaling processes, which was instigated by the experiments of Galvani $(\sim 1790)$. He demonstrated that static electricity and lightning made frog legs twitch. Galvani's experiments inspired later writers and cinematographers to focus on electrodynamic processes as relevant to consciousness.

The story of Frankenstein by Mary Shelley (1818) is a prime example of such thinking. Here, a human corpse was animated by electrical spark, a la Galvani's experiments with frogs. This theme has captivated the popular mind for more than 200 years, witness the many books and more than 50 movies themed on the Frankentein character. Today's genre of such an idea is exemplified by movies such as Blade Runner and ExMachina though they never explain why a robotic device would desire to be free or to be like a human. How could such desire be programmed?

Expanding on the electrodynamic models of Galvani (1780) and later Hebb (Hebb, 1949), experiments with EEG studies and transcranial activation of brain regions as well as electroshock therapy continue this approach (Frey 1993; Piccolino et al, 1997; Fecteau et al, 2007).

The realizations that chemical processes also affect consciousness came much later and was more deliberate. Initially, acetylcholine, a neurotransmitters (NT) and its receptor (Tansey 1991; Changeux, 2012, 2017), were identified as key features of neural activation related to consciousness. Other NTs and cognate receptors were also found to be capable of activating body organs and tissues, simultaneous to inducing emotive states (Table 1). The proposal that a chemical description of mentality was appropriate came much later (Chang, 2017). The graphic timeline (Figure 2) highlights the historical milestones in the neuroscientific identification of each signaling mode. 


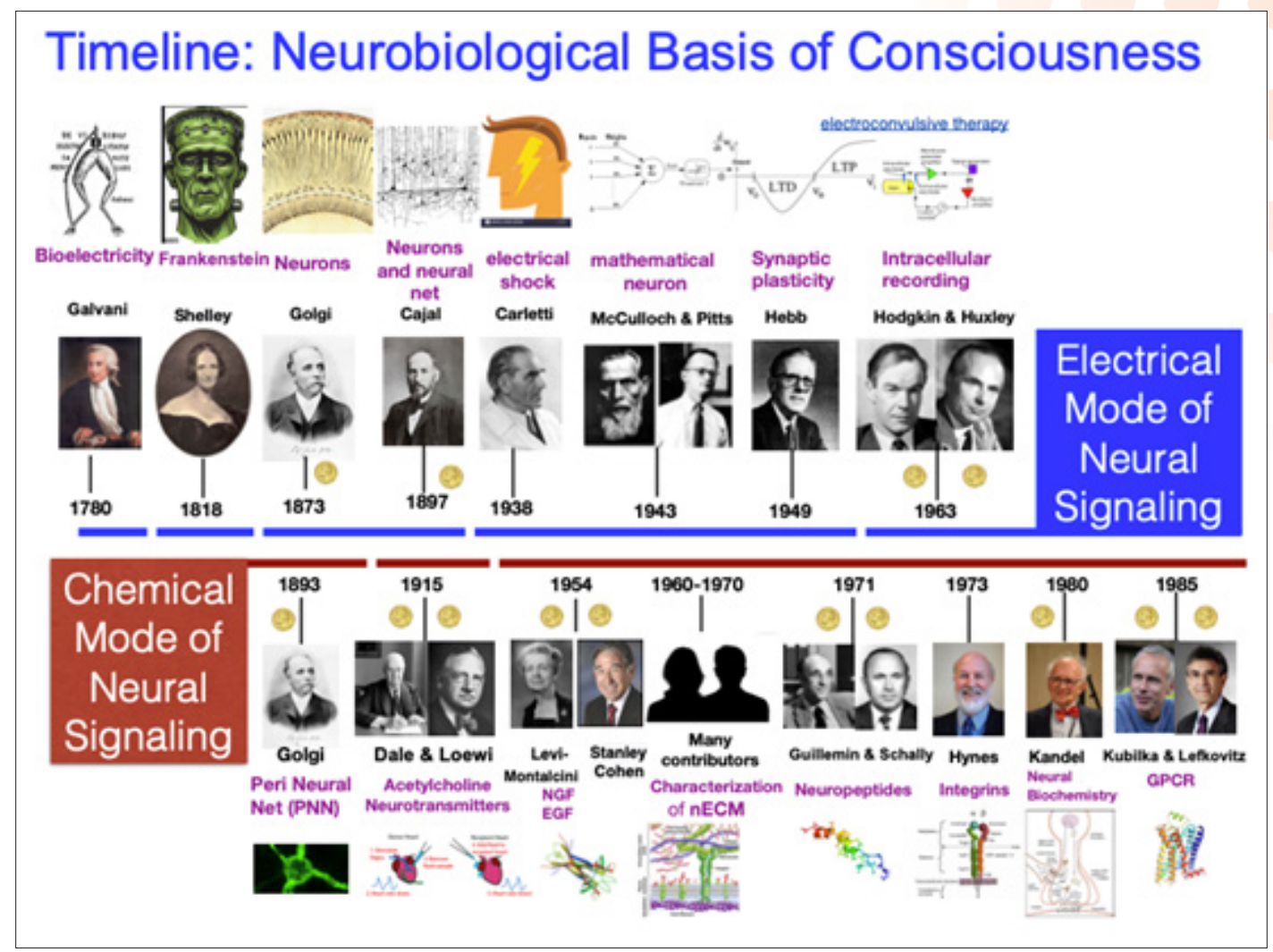

Figure 2: Timeline in the sequence of concepts and discoveries of electrodynamic and chemodynamic signaling modes related to the rationalization of consciousness.

\section{Discussion}

Our own contribution focused on a chemical approach to emotive coding of memory, as elaborated below (Marx \& Gilon, 2012-2020). The chemical approach to cognitive processes is buttressed by the abilities of psychoactive drugs to modulate mental states. We opine that a conception of neural consciousness must include chemodynamic, as well as electrodynamic descriptions.

As an aide to continuing this discourse, we clarify key terms, namely:

Feelings: perceptions of sensory stimuli associated with psychic states instigated by neurotransmitters (NTs). These do not require memory and are experienced by even the most primitive cells.

Emotions: Remembered feelings that require memory as well as a nervous system.

Memory: The storage and replay of information encoded in binary format as in computer chips. Note that computer memory is "demotive", lacking in an emotive quality that establishes the worth or value of any input.

Emotive memory: The recall of past stimuli experienced by the neural net. Its potency is characterized by subjective states. Code: The process of "coding" as applied to computers, has enriched the conception of neural memory and considerably broadened the scope of neural memory research (Gardner, 1987; Marx \& Gilon, 2016). Of course, the physical (sic. chemical) character of the encoding effectors must reflect the processes by which they operate.

Neural network: This term has been appropriated by the proponents of Artificial Intelligence (AI) to refer to microprocessors with many hardwired connections, though these do not at all mimic the indirect contacts between biological neural nets (Service, 2014; Pastur et al, 2016). Neuroscientists: employ the term to relate to biologically viable groups of communicating neurons which transmit chemical and electric signals through synaptic (Cajal, 1911; Sotelo, 2010; Koch et al, 2010) and ephaptic contacts (Vizi et al, 2010; Anastassiou et al, 2011).

The traits most commonly ascribed to consciousness (awareness, cognition, and mentation) relate to memory and emotions. Consider that without emotive qualifiers, the neural net could not value input stimuli, it would not long survive; its consciousness would be moot.

\section{Tripartite Mechanism of Neural Memory}

Our approach to emotions and neural memory is to describe them chemically. Essentially, we have proposed that the unit of cognitive information (cuinfo) is realized materially as a chemically based code which achieves an emotive state instigated by neurotransmitters (NTs) (Figure 3) (Marx \& Gilon, 2012-2020). 


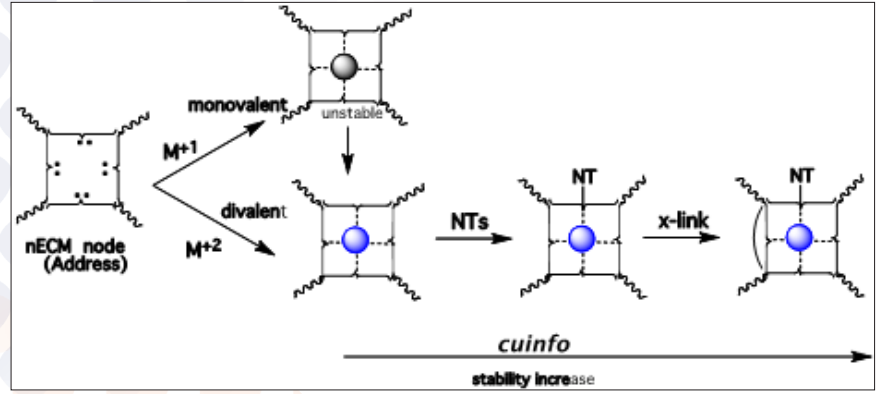

Figure 3: Tripartite mechanism. The formation of cuinfo complexes with different monovalent and polyvalent metal cations, as well as with different neurotransmitters (NTs), are all rendered more stable by crosslinking. The NTs impart an emotive quality to the cuinfo.

The tripartite mechanism of emotive memory elaborates on the individual (quantal) memory units (cuinfo) that can be processed by the individual neuron. But comprehensive memory results not from the cuinfo of an individual neuron, but from the collective activity of a group of neurons. Thus, a working model of how the brain generates emotive memory needs to meld physiologic effects with biochemical processes. In the tripartite model of emotive memory, the individual neuron is surrounded by cuinfo complexes within the nECM which it "reads" (Figure 3). The neural net integrates the contribution of the individual neurons into comprehensive recall (Agnati \& Fuxe, 2014).

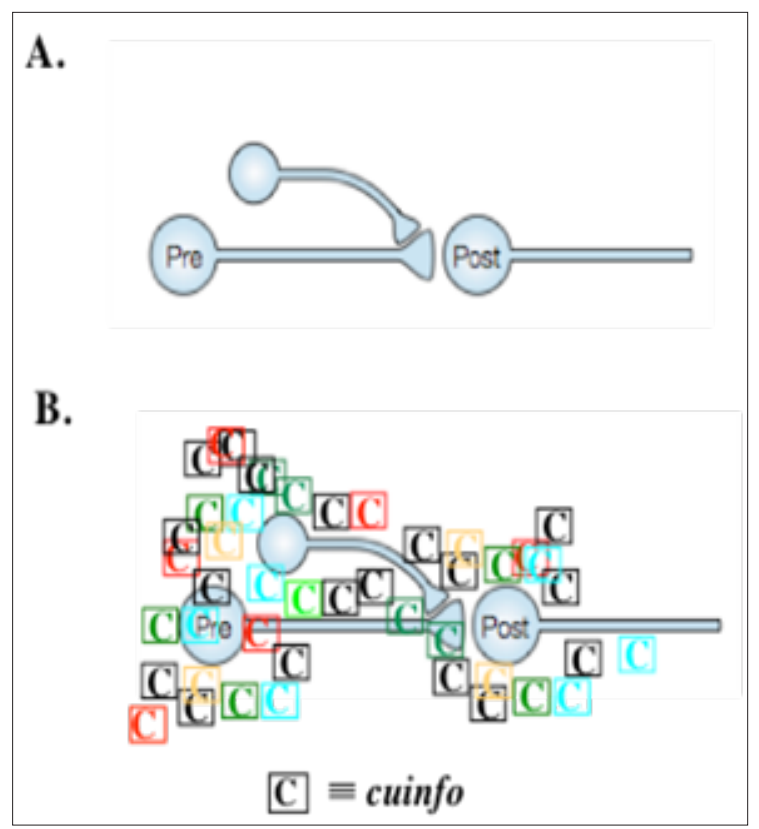

Figure 4: A chemographic schematic of A. a set of three naked neuron

B. the set of three neurons surrounded by cognitive units of information (cuinfo, C) within matrix of nECM (Marx \& Gilon, 2012-2020), which the neural net consolidates and transcends into conscious emotive memory (see tripartite mechanism).
We have previously described a tripartite mechanism by which neurons encode and decode (sic. write/read) the cuinfo (Marx \& Gilon, 2018). Essentially, this process (Figure 1, Equ. 1) is employed by neurons to chemically encode emotions linked to memory (see also Jeffreys, 1995). But communications between neurons also involves electrodynamic signaling.

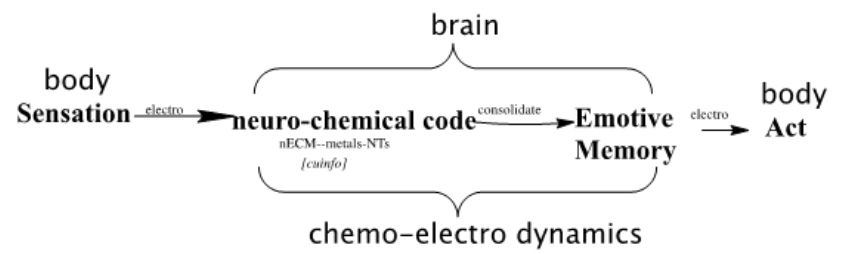

The philosopher asks: How is it possible for mental talent to be generated by the physical world?

Metaphysics is that branch of philosophy that examines the relationship between the physics of matter and mind.

Consider "metachemistry" as that branch of chemistry that examines how mental states are instigated by chemical processes experienced by neural nets.

Consciousness is a unique phenomenon distinct from the 4 basic forces of physics, namely gravity, electromagnetism, weak and strong nuclear interactions. Rather, consciousness and emotive memory are facets of another force, the life force that animates neural nets. Consciousness is a force of metabolic energy, phase changed into existential dimensions which emerged from the increasing complexity of neural interactions (Figure 5).

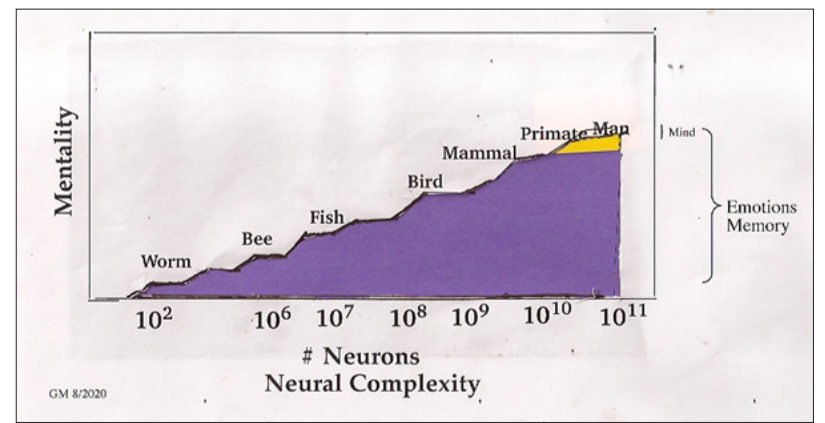

Figure 5: The emergence of conscious mental states, such as emotions and memory, correlates with the evolving size and complexity of neural net signaling (Mattews, 2001; Roth \& Dicke, 2005).).

The example of biology suggests that a minimum of 300 neurons (i.e. C. elegans) are required to achieve the "criticality"(Carhart-Harris, 2018) of the emergence of a mental talent, namely conscious emotive memory, from a neural net (Figure 4).

In spite of the known effects of natural and synthetic drugs, most expositions of consciousness do not consider psychochemical processes, but describe electro-dynamics. The drug approach to neural memory has been skewed to pharmacology, which does not address how neural nets emote or remember, but focuses on the gross physiologic and behavioral effects of 
drugs. Nonetheless, the effects of psycho-active drugs suggest that in addition to electrodynamic processes, consciousness must be chemically-modulated.

\section{Conclusion}

Computation has been fantasized as being able to mimic the workings of the brain (Bosse et al, 2014). Recall the titles of papers from early computer pioneers and their progeny: The Laws of Thought, Boole,1853; Computing Machinery and Intelligence Turing 1950 ; Artificial Minds, Franklin, 1995; Neural Turing Machines Graves et al, 2014 ; Modelisation des mecanismes emotionnels pour un robot autonome, Hasson C. (2011); Neisser, 1963, The imitation of man by machine; Russel \& Norvig, 2009, Artificial Intelligence; Valverdu \& Casacuberta, 2009, Handbook of Research on Synthetic Emotions and Sociable Robotics:

The consciousness involving emotions and memory is fueled by the metabolic energy expended by neurons. One could consider that the biological neural net transcends metabolic energy into new a dimension of existential consciousness not described by the metrics of physics or the algorithms of Artificial Intelligence (Ullman 2019). Notwithstanding, the identification of the nECM combined with NTs and metal cations as components of neural memory suggests that such components might be employed to construct synthetic memory devices, as an alternative to silicon-based chips (Prime \& Paul, 2009).

For neurobiology, we expect that combining the efforts of electro-dynamic neuroscience with chemical approaches to neural sensors (i.e. Agnati \& Fuxe, 2014; Chang, 2017) will eventually clarify the causative mechanism of emotive memory from which the experience of consciousness emerges.

\section{Acknowledgement:}

(By GM). A memorium to my late wife, the artist Georgette Batlle (1940-2009). Thanks to my daughter Danae (Jerusalem) and son Jonathan (Weehawken, NJ), for warm encouragement and practical support.

\section{Conflict of Interest}

GM is a founder of MX Biotech Ltd., with the commercial goal of developing new classes of "memory materials" and "memory devices".

CG is emeritus professor at the Institute of Chemistry, The Hebrew University of Jerusalem. He is active in developing technologies for the synthesis and conversion of peptide analogues of active regions of proteins into orally available drugs.

Notwithstanding, the ideas forwarded here are scientifically genuine and presented in good faith, without commercial clouding of the concepts expressed here.

\section{References}

The citation presented here are only the tip of the iceberg. Many more experimentalists and theoreticians have worked on cognition and memory, more than we could cite here. Nevertheless, we salute them.

1. Agnati, L.F., Fuxe, K (2014) Extracellular-vesicle type of volume transmission and tunnelling-nanotube type of wiring transmission add a new dimension to brain neuroglial networks. Philos. Trans. R Soc. Lond. B Biol Sci. 369(1652). pii: 20130505.

2. Aho, A.V (2011) What is Computation? Computation and computational thinking. Ubiquity, an ACM publication, January, 2011

3. Albrecht, A., Redavide, E., Regev-Tsur, S., Stork, O. \& Richter-Levin, G (2021) Hippocampal GABAergic interneurons and their co-localized neuropeptides in stress vulnerability and resilience, Neuroscience and Biobehavioral Reviews 122: 229-244.

4. Anastassiou, C.A., Perin, R., Markram, H. \& Koch, C (2011) Ephaptic coupling of cortical neurons. Nature Neuroscience 14: 217-223.

5. Arbib. M.A. (1987) Brains, Machines and Mathematics. ( $2^{\text {nd }}$ Ed). Springer Verlag, Berlin.

6. Arbib, MA. (2000) Warren McCulloch's Search for the Logic of the Nervous System, Perspectives in Biology and Medicine 43: 193-216.

7. Atkinson, R.C., \& Shiffrin, R.M. (1965) Mathematical models for memory and learning. Technical report 79 . Institute for Mathematical Studies in Social Sciences. Stanford University, CA.

8. Bosse T., Broekens J., Dias J. \& van der Zwaan J. (Eds.) (2014) Emotion Modeling. Towards Pragmatic Computational Models of Affective Processes. Springer, New York.

- a.Hudlicka, E., From habits to standards: Towards systematic design of emotion models and affective architecture. pp 3-23.

- Dastani,, M., Floor C. \& Meyer, J.J., Programming agents with emotions. pp 57-75.

- Lowem R. \& Kiryazovm K., Utilizing emotions in autonomous robots: An enactive approach. pp 76-98.

9. Boole, G. (1853) The Laws of Thought. The Mathematical Theories of Logic and Probabilities. Project Gutenberg (EBook \#15114); Released: Feb. 2005.

10. Carhart-Harris, R, Roseman, L., Haijen, E., Erritzoe, D., Watts, R., Branchi, I. \& Kaelen M. (2018) Psychedelics and the essential importance of context. Psychopharmacol. 32: 725-731.

11. Chalmers, D.J. (1995) The puzzle of conscious experience. Sci. Amer. December

12. Chang, C.J. (2017) Bioinorganic life and neural activity: Toward a chemistry of consciousness? Acc. Chem. Res. 50: 535-538.

13. Changeux, J.P. (2010) Allosteric receptors: From electric organ to cognition. Annu. Rev. Pharmacol. Toxicol. 50: $1-38$.

14. Changeux, J.P.(2012) The concept of allosteric modulation: 
an overview. Drug Discov. Today 10: e223-e228.

15. Changeux, J.P. (2012) Conscious processing: Implications for general anesthesia. Curr. Opin. Anaesthesiol. 25(4): 397-404.

16. Changeux, J.P. (2017) Climbing brain levels of organisation from genes to consciousness. Trends in Cognitive Sciences 21: 168-181.

17. Changeux, J.P. (2018) The nicotinic acetylcholine receptor: a typical 'allosteric machine'Phil. Trans. R. Soc. B 373: 2017017420170174

18. Changeux, J.P. \& Lou, H.C. (2100) Emergent pharmacology of conscious experience: new perspectives in substance addiction. FASEB Journal 25: 2098-2108.

19. Churchland, P. (2013) Matter and Consciousness. MIT Press, Cambridge, MA.

20. Cleeremans, A. (2008) Consciousness: The radical plasticity theory. Prog. Brain Res. 168: 19-33.

21. Dehaene, S., Kerszberg, M., \& Changeux, J.P. (1998) A neuronal model of a global workspace in effortful cognitive tasks, Proc. Natl. Acad. Sci. USA 95: 14529-14534.

22. Dehaene S. \& Changeux J.P. (2011) Experimental and theoretical approaches to conscious processing. Neuron 70: 200-227.

23. D'Esposito, M. (2007) From cognitive to neural models of working memory. Phil. Trans. R. Soc. B 362 : 761-772.

24. Edelman G. \& Tononi G. (2000) A Universe of Consciousness. Basic Books, New York.

25. Freeman, W.J. (1997) Three centuries of category errors in studies of the neural basis of consciousness and intentionality. Neural Netw. 10: 1175-1183.

26. Frey, A.H. (1993) Electromagnetic field interactions with biological systems. FASEB J. 7: 272-281.

27. Fecteau, S., Pascual-Leone, A., Zaldm, D.H., Liguori, P., Théoret, H., Boggio, P.S. . (2007) Activation of prefrontal cortex by transcranial direct current stimulation reduces appetite for risk during ambiguous decision making, $J$. Neurosci. 27: 6212-6218.

28. Franklin S. (1995) Artificial Minds. MIT Press, Cambridge, MA.

29. Gallistel, C.R., \& King A.P. (2009) Memory and the Computational Brain. New York: Wiley Blackwell, 2009.

30. Gardner, H. (1987) The Mind's New Science. A History of the Cognitive Revolution. Basic Books, New York.

31. Gerbaulet, J.-P., Henry, M. (2019) The 'ConsciousnessBrain' relationship. Substantia 3: 113-118.

32. Graves, A., Wayne G. \& Danihelka I. (2014) Neural Turing Machines Google DeepMind, London, UK arXiv: $1410.5401 \mathrm{v} 2$ [cs.NE]

33. Guidolin. D., Albertin. G., Guescini. M., Fuxe. K. \& Agnati L.F. (2011) Central nervous system and computation. Quart. Rev. Biol. 86: 265-85.

34. Hameroff, S. \& Penrose, R. (2014) Consciousness in the Universe: A review of the 'OrchOR' theory. Physics of Life Reviews 11: 39-78.

35. Hasson C. (2011) Modelisation des mecanismes emotionnels pour un robot autonome: perspective developpementale et sociale. $\mathrm{PhD}$ Thesis, Universite de Cergy Pontoise, France.
36. Hebb, D.O. (1949) The Organization of Behavior. Wiley, New York.

37. Hörmera, B.A., Vermaa, D., Gassera, E, WieselthalerHölzla, A., Herzog, H. \&Tasana, R.O. (2018) Hippocampal NPY Y2 receptors modulate memory depending on emotional valence and time. Neuropharmacology 143: 20-28.

38. Howard, N. \& Guidere, M. (2012) LXIO: The mood detection Robopsych. The Brain Sciences Journal 1: 7177.

39. Jeffress, L.A., (1951) Cerebral Mechanisms in Behavior. The Hixon 1947 Symposium. Wiley, New York.

40. Jefferys, J.G. (1995) Nonsynaptic modulation of neuronal activity in the brain: Electric currents and extracellular ions. Physiol. Rev. 75: 689-723.

41. Jong, Y.I., Harmon, S.K, \& O’Malley, K.L. (2018) Intracellular GPCRs Play Key Roles in Synaptic Plasticity, ACS Chem. Neurosci. 9: 2162-2172.

42. Koch, C., Massimini, M., Boly, M. \& Tononi, G. (2016) Neural correlates of consciousness: Progress and problems. Nat. Rev. Neurosci 17: 307-321.

43. Lange, M. (2002) The Philosophy of Physics: Locality, Fields, Energy, and Mass. Blackwell Publishing, London.

44. Longuet-Higgins, H.C. (1981) Artificial intelligence--a new theoretical psychology? Cognition 10: 197-201.

45. Marx G. \& Gilon C. (2012) The molecular basis of memory. ACS Chemical Neurosci. 3: 633-642.

46. Marx G. \& Gilon C. (2013) The molecular basis of memory. MBM Pt 2: The chemistry of the tripartite mechanism. ACS Chemical Neurosci. 4: 983-993.

47. Marx, G. \& Gilon C. (2016) The molecular basis of neural memory. MBM Pt 6: Chemical coding of logical and emotive modes. Int. J. Neurology Res. 2: 259-268. doi: $10.17554 / \mathrm{j}$.

48. Marx G. \& Gilon C (2018) The molecular basis of neural memory. Part 10. The sins and redemption of neurobiology. J. Neurol. Neurocrit. Care 1: 1-7.

49. Marx G. \& Gilon C. (2019 The tripartite mechanism as the basis for a biochemical memory engram. J. Integr. Neurosci. 18: 181-185. doi: 10.31083/j.jin.2019.02.6101.

50. Marx G. \& Gilon C. (2020)

Interpreting neural morphology. Acta Scientific Neurology 3: 1-4.

- Tripartite mechanism of neural memory: Proof-of-concept with neuromimetic impedence electrodes. Biomedical Research and Clinical Review 1(3)-021

- The molecular basis of neural memory. Part 11. Chemelectric write /read processes. J. Neurosurg. Imaging Techniques 6: 283-301.

- Quantum considerations of neural memory. In: Quantum Boundaries of Life, Vol. 82, Poznanski R.R. \& Branda E. (Eds), Elsevier, Amsterdam.

- Encoding neural memory. Front Drug Chem. Clin. Res. doi: 10.15761/FDCCR.1000149

- Tripartite mechanism of neural memory: Proof-of-concept with neuromimetic impedance electrodes. Biomedical Research and Clinical Review 1(3)-021.

51. Matson, W.I. (1971) Spinoza's Theory of Mind, The 
Monist 55: 567-578, https://doi.org/10.5840

52. Matthews, G.G. (2001) Evolution of Nervous Systems. Neurobiology: Molecules, Cells, and Systems. WileyBlackwell. p. 21. ISBN 978-0-632-04496-2.

53. McCulloch, W.S. \& Pitts W.H. (1943) A logical calculus of the ideas immanent in nervous activity. Bulletin of Mathematical Biophysics 7, 115-133. Reprinted in McCulloch 1964, pp. 16-39.

54. Merolla, P.A., Arthur J.V., Alvarez-Icaza, R., Cassidy, A.S., Sawada, J., Akopyan, F., Jackson, B.L., Imam, N., Guo, C., Nakamura, Y., Brezzo, B., Vo, I., Esser, S.K., Appuswamy, R., Taba, B., Amir, A., Flickner, M.D., Risk, W.P., Manohar, R. \& Modha. D.S. (2014) A million spiking-neuron integrated circuit with a scalable communication network and interface. Science 345: 668673.

55. Morris, C.W. (1932) Six Theories of Mind University Chicago Press, Chicago Ill.

56. Pastur-Romay, L.A., Cedrón, F., Pazos, A. \& Porto-Pazos, A.B. (2016) Deep artificial neural networks (ANN) and neuromorphic chips for big data analysis: Pharmaceutical and bioinformatics applications. Int. J. Molecular Sciences 17: 1313-1339.

57. Piccolino, M. (1997) Luigi Galvani and animal electricity: Two centuries after the foundation of electrophysiology. Trends in Neurosciences 20: 443-448.

58. Pockett, S. (2014) Problems with theories that equate consciousness with information or information processing. Front. Syst. Neurosci. 8: 1-3.

59. Prentner, R. (2017) Consciousness: A molecular perspective. Philosophies 2: 26-32.

60. Prime D. \& Paul S. (20090 Overview of organic memory devices. Philos.Transact. R. Society A 367: 4141-4157.

61. Rosenblum B. and Kuttner F. (2011) Quantum Enigma: Physics Encounters Consciousness. 2 ${ }^{\text {nd }}$ Ed. Oxford University Press, Cambridge, UK.

62. Roth, G. \& Dicke, U. (2005) Evolution of the brain. Trends Cogn. Sci. 9: 250-257.

63. Russel, S. \& Norvig P. (2009) Artificial Intelligence : A Modern Approach. $3^{\text {rd }}$ Ed. Pearson Publishers, NY.

64. Ryle G. (1949) The Concept of Mind. Penguin Books, UK.

65. Semon, R. (1923) Mnemic Psychology G. Allen \& Unwin, London.

66. Shelley M. (1818) Frankenstein. Oxford University Press, World's Classics, 1998, New York.

67. Senkov, O., Andjus, P., Radenovic, L., Soriano, E. \& Dityatev, A. (2014) Neural ECM molecules in synaptic plasticity, learning and memory.In. In: Dityatev A, WehrleHaller B, Pitkänen A. (Eds), Progress in Brain Research 214: Ch 3, Amsterdam.

68. Sejnowski, T.J. (2015) Consciousness. Daedalus 144: 123-132. doi: 10.1162/DAED_a_00321.

69. Service, R.F. (2014) Cover image and story: The brain chip. Science 345: 614-616.

70. Shrodinger, E. (1958) Mind and Matter. Reprinted in: What is Life.1967. Cambridge University Press, Edinburgh, UK

71. Skirry, J. (2016) Rene Descartes: The Mind-Body Distinction. Internet Encyclopedia of Philosophy. ISSN
2161-0002.

72. Sloman, A. (2008) Kantian philosophy of mathematics and young robots. Proceedings $7^{\text {th }}$ International Conference on Mathematical Knowledge Management Birmingham, UK, 28-30 July 2008.

73. Sorli, A., Fiscaletti, D. \& Mageshwaran, M. (2016) Advanced Relativity: Unification of space, matter and consciousness. Neuro Quantalogy 14: 645-656.

74. Sotelo, C. (2011) Camillo Golgi and Santiago Ramon y Cajal: The anatomical organization of the cortex of the cerebellum. Can the neurondoctrine still support our actual knowledge on the cerebellar structural arrangement? Brain Res. Rev. 66: 16-34.

75. Tansey, E.M. (1991) Chemical neurotransmission in the autonomic nervous system: Sir Henry Dale and acetylcholine. Clin. Auton. Res. 1: 63-72.

76. Tuszynski. J. (Ed) (2006) The Emerging Physics of Consciousness. Springer, New York

77. Ullman, S. (2019) Using neuroscience to develop artificial intelligence. Science 363: 692-693.

78. Valverdu, J. \& Casacuberta, D. (2009) Handbook of Research on Synthetic Emotions and Sociable Robotics: New Applications in Affective Computing and Artificial Intelligence. Information Science Reference, Hershey, NY. 2009

79. Von Neumann, J. (1950) Collected works, Taub (Ed). The Natural and Logical Theory of Automata. Volume V Design of computers, theory of automata and numerical analysis. Pergamon Press, New York.

80. Von Neumann, J. (1951 The Cerebral and Logical Theory of Automata. In: The Hixon 1947 Symposium, Jeffress, L.A. (Ed) Wiley, New York.

81. Vizi, E.S., Fekete, A., Karoly, R. \& Mike, A. (2010) Nonsynaptic receptors and transporters involved in brain functions and targets of drug treatment. Brit. J. Pharmacol. 160: 785-809.

82. Yin. B. \& Troger A. (2011) Exploring the 4th dimension: Hippocampus, time and memory revisited. Front. Integrative Neuroscience doi: 10.3389/front.2011.00036

Copyright: (C2021 Gerard Marx. This is an open-access article distributed under the terms of the Creative Commons Attribution License, which permits unrestricted use, distribution, and reproduction in anymedium, provided the original author and source are credited. 